\title{
Estructura de la política nacional de ciencia y tecnología
}

\section{Principios de política}

Los principios básicos que guiarán la política serán los siguientes:

- Un pais orientado hacia la calidad total que integra competitividad y productividad.

- Un enfoque hacia la sostenibilidad y respeto por el medio ambiente y recursos naturales

- La concertación como eje articulador de la viabilidad de la Nación.

- La búsqueda de la mejora constante del nivel de vida de la población

- La popularización y difusión del ciencia y tecnología como guía operativa

- La valorización del conocimiento y su pronta aplicación

- La priorización hacia la incorporación de la micro y pequeña empresas en el esfuerzo nacional de calidad.

- Horizonte nacional

II. Bases de política

1) Breve caracterización de El Salvador: diagnóstico sobre ciencia y tecnología

- A partir de 1950 el avance cientifico y tecnológico mundial ha transformado de manera sorprendente y hasta radical las tecnologfas clásicas convencionales. En la actualidad hay continuos oleajes de cambios tecnicos, los mercados son renovados periódicamente por nuevos bienes y servicios de elevado contenido tecnológico, habiendose achicado significativamente la duración del ciclo inventivo: ciencia y 
tecnologfa y sus materializaciones prácticas en bienes y servicios.

Esta situación es completamente diferente a lo acontecido en la Primera y Segunda Revolución Industrial (1750-1850 y 1850-1930) que impactaron esencialmente sólo a la industria manufacturera.

- Este desarrollo científico y tecnológico ha transformado simultánea y aceleradamente el marco internacional hacia un proceso de globalización intensivo y con grandes fuerzas arrolladoras, proceso que representa grandes retos para los parses subdesarrollados en general, con mayor fuerza para aquellos que no han adoptado para sf o tomado con seriedad dicho proceso y no poseen una visión más o menos adecuada con un horizonte de al menos 10-15 affos. La revolución cientifico-tecnologica actual es justamente una verdadera revolución y condiciona e impacta la economfa, sociedad, organizaciones empresariales y gremiales, aparato estatal, sistema educativo, vida familiar y vínculos funcionales y conductuales.

- En julio de 1992 se crea el Consejo Nacional de Ciencia y Tecnología (CONACYT) que vino a constituir un gran paso en el mejoramiento e institucionalidad de los esfuerzos y acciones de ciencia y tecnologa absorbiendo labores del desaparecido Centro Nacional de Productividad (CENAP), Departamento de Ciencia y Tecnología del ex-MIPLAN y labores de metrologra y normalización del Ministerio de Economfa. El Consejo que pese a sus limitados recursos ha realizado una labor muy aceptable, sobre todo si se considera el nuevo entomo socioeconómico del pars, el empuje dol proceso de globalización a nivel internacional y la ausencia de instrumentos básicos.

No obstante, el engranaje institucional es todavía muy débil y hay carencia de instrumentos fundamentales que permitan dar un salto cualitativo y mejorar la gestión y la articulación con el resto del aparato estatal, universidades e institutos tecnologicos, gremios profesionales, empresa privada, sistema educativo básico y medio, y núcleos familiares.

- Sin menoscabo de los esfuerzos realizados se podría decir que no existe en un grado aceptable una política de ciencia y tecnología en el país. La variable ciencia y tecnología virtualmente representa una variable residual o secundaria. Hasta el presente El Salvador ha sido un importador neto de ciencia y tecnología, un mero adaptador de procesos, usuario neutral y virtual espectador de la revolución cientúfico-tecnologica en marcha a nivel planetario.

- Los recursos naturales y la contaminación en sus diversas formas muestran fuertes índices de deterioro lo que ha conducido a pérdidas significativas y a veces irreversibles en el suelo, aguas subterráneas, derrumbes, azolvamiento de presas hidroeléctricas, disminución de caudales de rfos, extinción de especies animales y vegetales, agotamiento de recursos costeros, cambios climáticos y otros. Esta situación en realidad es bastante dramática y se vuelve sombría si se considera el fuerte crecimiento poblacional y la insuficiente capacidad regulatoria del Estado.

- En el sistema financiero público no existe al menos una línea de crédito básica para financiar proyectos y actividades científico-tecnologicas ni tam- 
poco un fondo de desarrollo cientifico y tecnologico que promueva la investigación básica y aplicada, y estimule la creatividad y formación de cuadros humanos. El sistema financiero privado, por su lado está muy lejos de dar soporte financiero para los proyectos y actividades en referencia, y la ciencia y tecnología constituye más bien una frase exótica en el mercado e ingeniería financiera. De hecho, la lógica de la ciencia y tecnología no está en la agenda del sistema financiero y mucho menos en la agenda pública.

- Los diversos niveles de la educación en El Salvador evidencian índices cualitativos alarmantes y la enseñanza de la ciencia y tecnología es marginal y no se estimula la vocación ni la creatividad en los educandos. La calidad de la educación, su pertinencia y dinámica constituyen un fuerte valladar para la formacion de recursos humanos. La infraestructura existente para evaluar la enseñanza y la experimentación en ciencia es obsoleta o insuficiente, ya se trate de profesores, equipo, laboratorios, bibliotecas, materiales, acceso a información técnica especializada, aplicación del método científico y administración.

- Las Universidades e Institutos Tecnol6gicos en general evidencian una marcada debilidad en infraestructura de investigación, limitada difusión de los resultados de investigacion, y no hay especialidades en campos fundamentales de la ciencia y tecnología.

- Los mecanismo de enlace universidad-empresa son virtualmente débiles, la enseñanza y formación está bastante divorciada del mercado y de las tendencias del mundo productivo empresarial, la divulgación de los resultados de investigacion es muy limitada, y la empresa privada de ordinario es muy tradicionalista y prefiere ser una importadora neta de tecnología. Parecieran prevalecer "ćŕrculos viciosos y la inercia de las barreras culturales, administrativas, y de enfoque y desconfianza", según los cuales todos tienen razon en sus planteamientos sectoriales.

En la búsqueda de razones explicativas y en rigor, la insuficiente competencia, la débil cultura de la innovación y la calidad y el no saber trabajar en equipos con objetivos compartidos, se consideran mucho más determinantes vis a vis el carácter familiar empresarial, dimensión operacional y las barreras ideologicas.

- A nivel formal y en diversos círculos tiende a sostenerse que la mayor riqueza del país es su gente, sus recursos humanos, especialmente la clase trabajadora, cuyo empuje y laboriosidad es puesta de ejemplo también en el exterior. Sin ignorar la validez histórica y contextual de dicha "tesis", debe señalarse que ella en un proceso de desarrollo es muy relativa. La mano de obra barata, abundante y como ventaja comparativa estática ya no basta y presenta sus propios estrangulamientos. 
En el mundo contemporáneo, la calidad, destrezas y conocimientos de la mano de obra constituyen un imperativo para que ésta sea una aliada de la innovación empresarial. No obstante, la concepción sobre su papel y los programas de capacitación, en la práctica, siguen siendo tradicionalistas, con predominio de oferta y de aprovechamiento de fondos que hay que gastar.

- Las diversas redes de información científica y tecnológica son muy formales y hasta cerradas, y pareciera existir una mentalidad de dispersión y fragmentación de esfuerzos, aun en redes importantes como las de salud, educación, medio ambiente, alimentos y sector agropecuario. Las redes cualquiera sea su naturaleza y especificidad, deben ser justamente tales y convertirse en apoyos para la innovación y logro de ventajas competitivas, y superar el estancamiento que tienden a mostrar y perpetuar en tanto meras ventajas operacionales y confusión de fines y medios.

- El número de servicios que presta el Centro de Información Tecnológica (CIT) de CONACYT y su cobertura son muy limitados, y se debe reconocer que no existe la adecuada gestión, que permita vincular más y mejor los mismos con el sector empresarial en general. Este aspecto hay que tomarlo en serio para robustecer el papel de facilitador de CONACYT.

- La Red SV-NET ha costado implantarla a cabalidad en el país y de hecho El Salvador fue el último miembro en hacerlo en Centroamérica, a pesar de la ayuda de la OEA y donación de equipo. Más allá de las expectativas y entusiasmo iniciales, se debe reconocer que hace falta trabajar muy fino para aprovechar en buen grado este instrumento en los negocios en general, educación y micro y pequeña empresa en especial.

- Los indicadores de ciencia y tecnología existentes son parciales, cuasiirrelevantes y discontinuos, y usualmente son calculados por analistas interesados, por lo que para efectos prácticos, no se dispone de indicadores oficiales que revelen aspectos básicos del quehacer científico-tecnológico.

- Desde los años cincuenta, la normalización ha revolucionado muy poco en la actividad productiva nacional, a pesar de los intentos realizados, y más bien la cultura de la normalización, información adecuada al consumidor y la calidad en los sectores productivos es incipiente. No obstante, se advierten cambios de actitud, nuevas formas de pensar y mayor involucramiento empresarial.

- Hay vacíos importantes en la aplicación del Sistema Internacional de Unidades de Medida que es el sistema legal conforme a la Ley de CONACYT y que es recomendado por la Organización Mundial de Comercio (OMC). En efecto, en la vida cotidiana existe un caos en metrología y el consumidor es fácilmente engañado y no necesariamente debido a malicia o mala fe. 
- La demanda por cientificados de calidad ha sido mínima limitíndose a unas poses empresas exportadoras de productos no tradicionales y se adviente la falta de un proceso de acreditación de las empresas certificadoras.

- Si bien este campo es de apoyo y de soporte en la innovación y gestión de proyectos de ciencia y tecnología, también es relevante para la reconversión productiva, penetración y mejoramiento de mercados y como complemento de las acciones tendientes a la conformación de un sistema funcional de ciencia y tecnología y de introyección de la cultura de la ciencia tecnología e innovación.

- Los sectores más directamente involucrados con la ciencia y tecnología-Estado, universidades e institutos tecnológicos, empresa privada y gremios profesionales, no muestran una interrelación relativamente dinámica ni vasos comunicantes con convergencia de esfuerzos, lo cual refuerza la tendencia hacia una débil institucionalidad y a una limitada identificación con la cultura de la ciencia y tecnología.

- El diagnóstico revela que en El Salvador y por encima de los deseos formales a varios niveles e instancias técnico-administrativas, no existen las bases para una cultura de ciencia, tecnología e innovación. La concepción e identificación no están introyectadas al interior del aparato estatal, empresas, gremios, sistema educativo y núcleos familiares, aunque se advierten nuevas formas de pensar, de gestión en el proceso de ciencia y tecnología, una reforma educativa importante, cierta innovación tecnológica y perspectivas de perfeccionar e integrar mucho mejor el accionar y la irradicación de sus resultados.

- El panorama que revela el diagnóstico es de que la tarea es inmensa, de titanes pero muy desafiante. Por ende, hay que ser selectivo en el accionar, definir mejor las prioridades en estrecho amarre con la demanda y señales del mercado, y ser más pragmáticos en la gestión, vale decir entender y aplicar el rol normativo, regulatorio y de promoción del Estado en general y de CONACYT en particular.

La economía social de mercado exige mucha ciencia y tecnología y mística creativa e innovadora. 


\section{El pars que pretende la politten: la vibion de El Salvedor 2021 deade la peripectiva cientifica-teconolgejo}

\section{A) La colided de pairs en cifres: vision}

Indicador

1995

2021

(Proceso-meta)
Ingreso por habitante

Crecimiento económico

Exportaciones de bienes

Exportaciones de bienes/ PIB

Desempleo abierto

Inflación

Crecimiento de la Productividad

Carga tributtaria

Pobreza relativa

Pobreza absoluta

Gasto en ciencia y tecnologfa/ PIB

Crecimiento poblacional

Mortalidad

Analfabetismo

Cobertura educativa básica

Población económicamente activa

Esperanza de vida

$\begin{array}{rcr}\$ 736.5 & \text { No menor } & \$ 4.250 .0 \\ 5.7 \% & " & 6.5 .-7.0 \% \\ 8.0 \% & " & 14.0-16.0 \% \\ 30.0 \% & " & 50.0 \% \\ 40.0 \% & \text { No mayor } & 10.0 \% \\ 12.0 \% & " & 4.0-5.0 \% \\ 1.0 \% & \text { No menor } & 5.0 \% \\ 12.0 \% & \text { No menor } & 18.0 \% \\ 60.0 \% & \text { No mayor } & 20.0 \% \\ 35.0 \% & " & 0.0 \% \\ 0.2 \% & \text { No menor } & 1.5-2.0 \% \\ 2.3 \% & \text { No mayor } & 1.2 \% \\ 36.0 \% & " & 20.0 \% \\ 23.0 \% & " & 5.0 \% \\ 68.0 \% & \text { No menor } & 95.0 \% \\ 36.0 \% & " & 55.0 \% \\ 55 \text { años } & " & 72.0 \text { años }\end{array}$

Fuente: Universidad Centroamericana José Simeón Cafaas, UCA

En el cuadro anterior se puede advertir en forma sumaria la situación actual de El Salvador conforme a una gama de indicadores básicos, y la visión razonable que se vislumbra para el año meta 2021.

La concretización de dichas metas implica un esfuerzo sostenible en materia de política socioeconómica, liderazgo gubernamental, concurrencia de esfuerzos, logro de metas intermedias, confianza, estímulo al ahorro e inversión, alza continua en la productividad, y la adopción en el sistema empresarial de una política agresiva en ciencia, tecnología e innovación.

Los indicadores cuantitativos hay que verlos como ordenes de magnitud que apuntan a un proceso tendencial y de mutuo eslabonamiento, que presentan la necesidad de su apropiación por todos los responsables. 


\section{b) Los especen conlitativos de la volko}

En cuanto indicadores cualitativos la política nacional de ciencia y tecnología ofrece como visión el esplritu que tiene el legislador cuando aprueba la Ley de Creación del Consejo Nacional de Ciencia y Tecnología en 1992 respetando con ello la especificidad temática para la cuál ésta institución fue creada.

\section{En el año 2021 El Salviador}

a. Ha negociado el Tercer Programa Nacional de Desarrollo Cientifico y Tecnológico con los organismos financieros internacionales el cual ha contribuido ya significativamente a mejorar la infraestructura cientifica y tocnológica en los niveles empresarial, universitario y gubernamental propiciando de manera particular un ambiente de innovación, generación y transferencia de tecnología orientado de manera particular a la pequeña y mediana empresa.

b. Se tienen organizadas fundaciones de vinculación empresa-universidad cuyo objetivo es el de propiciar la adecuada gestión y transferencia tecnológica orientada a la pequeña y mediana empresa.

c. Se tiene organizado el registro del Sistema Nacional de Ciencia y Tecnología previsto en la Ley de Desarrollo Científico y Tecnológico y tanto las empresas públicas como privadas han reportado sus datos contando con indicadores de ciencia y tecnología que retroalimentan la política nacional debidamente, pues parte de las condiciones reales de los diferentes sectores.

d. Se ha logrado difundir de tal manera la cultura de la calidad que El Salvador ya no procede a elaborar norma salvadoreña, sino que homologa la normativa internacional por cuánto su interés es penetrar el mercado internacional y contar además con estandares internacionales que le permitan exigir alta calidad en las importaciones al país.

e. La empresa salvadoreña cuenta con su organización interna con departamentos o unidades especialidades en normalización, y se garantiza con ello la cultura de la normatización, útil para el abastecimiento del mercado interno y conquista de nuevos mercados exteriores, habiendo disminuido la brecha de calidad entre ambos.

f. El Salvador ha logrado en su esfuerzo por la normalización redefinir los terminos de su participación en el marco del mercado común centroamericano, negociación que ha permitido que las importaciones que hace el pars no sean vistas como una imposición de barreras no arancelarias por los países hermanos, pero que en lo fundamental le permite al país la suficiente flexibilidad para negociar con libertad en el marco internacional de acuerdo con sus propios ritmos de desartollo.

g. Todos los establecimientos educativos del pars hacen obligatoria la enseñan- 
za y aplicación del Sistema Internacional de Unidades (SI).

h. Se cuenta con un sistema integrado de normalización, metrología, verificación y cientificación de la calidad que en lo fundamental se apoya en los subsistemas nacionales de acreditación de laboratorios y centificación, verificación de la calidad y metrología legal e industrial a través del cual se aplica la legislación en lo relativo a la responsabilidad de la calidad, obligatoriedad de acreditar el cumplimiento de norma, certificación de calidad por lote, uso del sello de calidad; control metrológico, afericiones, tolerancias, calibración, uso de instrumentos de medida, fabricación, exportación, importación y venta de los instrumentos de medida, registro de productos envasados, laboratorios de prueba, ensayo y mediciones oficiales y privados; control de calidad de importaciones conforme a normas.

i. La organización del sistema de NMCC se ha desarrollado de tal manera que la ISO en Ginebra ya ha aceptado a El Salvador como miembro pleno de la organización sustituyendo el status por correspondencia que le caracterizaba desde enero de 1997.

j. El país cuenta con una excelente infraestructura de conectividad física para las comunicaciones electrónicas y para el desarrollo de inversiones en multimedia, habiéndose consolidado la apertura para la participación en un ambiente de alta competitividad a proveedores privados de conectividad física y a inversión extranjera de alta tecnología en multimedia.

k. El país ha consolidado la experiencia luego de más de veinte años de estar utilizando mecanismos de financiamiento apropiado para el desarrollo del sistema de Innovación y de Generación y Transferencia de Ciencia y Tecnología. El sector empresarial invierte en esta área a tasa de mercado dado que ha reducido significativamente los tiempos de la aplicación productiva de los instrumentos científicos tecnológicos.

1. El medio académico nacional es generador de profesionales y técnicos que están formados en la solución de problemas concretos que enfrenta la empresa salvadoreña incorporando su experiencia e impactando en los niveles de productividad, habiendo modificado... Por tanto la percepción desde el lado de la empresa respecto a la utilidad de su contratación.

m. El nivel técnico del país es altamente apreciado y remunerado por la sociedad habiéndose modificado sustancialmente la demanda a niveles de jefaturas intermedias dentro de las empresas, diferenciándose con claridad las aportaciones de los niveles académico y técnico.

n. El funcionario público concibe la inversión del sector privado como estratégica y en todo caso se siente como coinversionista. El éxito del empresario privado en su gestión representa el éxito del servidor público en propiciar un ambiente de desarrollo para la nación y por ende, altamente realizador. El 
respeto al servidor público y la alta confianza de la sociedad en la inversión del Estado marcan la diferencia entre El Salvador y buena parte de los países de América Latina.

o. Existen y funcionan a cabalidad un esquema de incentivos que promueven la modernización e innovación tecnológicas, especialmente financieros y fiscales.

p. Se han desarrollado alianzas estratégicas de empresas manufactureras en los servicios de clase mundial y El Salvador tienen empresas líderes mundiales instaladas en su territorio.

\section{A rea sociales y económicas de mayor potencialidad}

Si se consideran las áreas en que el país tienen potencialidades por sus ventajas comparativas (abundante mano de obra, clima laboriosidad, etc.) y competitivas (calidad, agrupamientos sectoriales, especialización, componentes tecnológico, ambiente de innovación, etc), así como de aquellas donde puede tener más posibilidades por el entorno mundial como ubicación geográfica, tendencia del desarrollo científico y tecnológico, mercado regional, etc., se proponen las siguientes áreas:

\section{a) Vinculadas con la producción y productividad}

Turística

Financiera

Agricultura intensiva para exportación

Acuicultura

Procesamiento de productos del mar

Agroindustria

Biotecnología

Industria de uso intensivo de mano de obra

Tecnología de alimentos

Nuevos materiales'

Estructuras metálicas

Química fina

Mecánica fina

Máquinas-herramientas

Microelectrónica

Procesos de ensamblaje en general

Informática y comunicaciones

b. Vinculadas con las condiciones necesarias y estabilidad sociopolítica

Producción y uso de nuevas fuentes de energía

Desarrollo de infraestructura vial, de comunicaciones, marítima y áerea. 
Ecosistemas y medio ambiente

Educación cientffica y tecnológica y capacitación orientada a la productividad.

Salud y seguridad social

Intermediación financiera

4. Condiciones y factores externos necesarios para garnethenr el alcance de visión de país

- Estabilidad sociopolítica

- Sistema educativo eficiente, eficaz y pertinente

- Régimen de salud y de seguridad social moderno y con cobertura cuasiuniversal

- Combate apropiado de la extrema pobreza

- Infraestructura pública de buen nivel y creciente

- Mano de obra capacitada y mística innovativa

- Aparato administrativo gubernamental moderno y eficaz on su papel de facilitador y normativo

- Aparato estatal impulsor y facilitador eficiente y eficaz de los prucesos productivos

- Intolerancia con los monopolios y oligopolios públicos y privedos, y cuando sea necesario estricta regulación y control

- Estimulo de la inversión extranjera directa

- Promoción del proceso de ahorro e inversión nacionales

- Flexibilidad y confianza en el mercado laboral

- Estudio permanente del entorno internacional

- Información pública de calidad, accesible y pertinente, y que sea justamente pública

- Fortalecimiento constente del sistema democrático, participación de la sociedad crvil y generación dinámica de consensos.

Tales factores en su conjunto y sus interrelación se consideran en realidad cuasiindispensables para el éxito en la ejecución de la política y la materialización de los objetivos y metas. 


\section{Marco global de política}

\section{Objetivos generales}

a. Inculcar una cultura e ciencia, tecnología e innovación en toda la sociedad y en particular en el sector empresarial.

b. Mejorar la institucionalidad, colaboración y capacidad persuasiva y motivadora de las acciones de ciencia y tecnología.

c. Fomentar la ciencia como bien público fundamental y la tecnología traducida en bienes y servicios privados.

d. Contribuir a crear e innovar el capital humano a todo nivel y en forma constante en particular en ciencia y tecnología.

e. Reforzar el ambiente de progreso, innovación y sostenibilidad que El Salvador necesita, conforme a la visión adoptada al año 2021 y profundo respeto por el medio ambiente y recursos naturales.

f. Elevar el potencial y creatividad práctica de los recursos humanos a través de la formación y capacitación para la productividad.

\section{Objetivos específicos}

a. Crear el Fondo de Desarrollo Científico y Tecnológico para apoyar la investigación científica y sus aplicaciones vinculadas a la demanda y la formación de cuadros humanos.

b. Ejecutar proyectos de impacto por año.

c. Promover la cultura de la ciencia y tecnología de El Salvador comenzando por la política nacional de Ciencia y Tecnología y su estrategia.

d. Fortalecer la infraestructura del ICT, Red SV-Net, red de laboratorios, el proceso de normalización y acreditación de la calidad y el capital humano de CONACYT.

e. Mejorar sostenida y significativamente la capacidad de gestión del proceso de ciencia y tecnología del paŕs.

f. Estimular la creatividad e inventiva de los salvadoreños, en particular en la educación básica, fuerza de trabajo y cuerpo gerencial.

\section{Alcance y temporalidad}

El alcance de la política será formalmente nacional pero funcional y en la práctica muy selectivo y concentrado en los componentes o ejes temáticos definidos.

El horizonte temporal de la política abarca hasta el año 2021, período adop- 
tado por el gobierno de la república en su programa de competividad, el cual proyecta 25 años, lapso adecuado para la política y la materialización de los objetivos establecidos.

En ese período se contará con infraestructura científico-tecnológica de primer nivel, avances apreciables en el capital humano, incrustación y promoción del país en el mundo, a estímulo al proceso de ahorro e inversión, promoción de la creatividad e inventiva nacionales, y el funcionamiento lo más pleno posible de los ejes temáticos de la política, y la operatividad del sistema nacional de ciencia, tecnología e innovación con autoimpulso y autoconvencimiento por los diversos actores y agentes políticos y socioeconómicos.

Los objetivos generales y especificos establecidos, se desarrollertn en detalle en las siguientes fases:

\section{A corto plazo 1996-1999:}

A corto plazo 1996-1999: se fortalecerá los servicios cientuficos tecnológicos y todo lo que ayude a las empresas a ser más productivas y competitivas; se desarrollarán al menos los siguientes fondos: i) De desarrollo tecnologico orientado a empresas e innovación, ajustado al cliente y a las necesidades de los proyectos de las empresas. ii) De capacitación de recursos humanos, para formar recursos, vinculado a planes institucionales de universidades y otras entidades que necesiten recursos competentes, para obtener mínimo de competencia generalizada (centros especializados). iii) De formación de masas criticas en el país, en base a sectores prioritarios en que se quiera tener más capacidad tecnológica o una concentración masiva y especializada de científicos. iv) Para servicios científicos tecnológicos.

Adicionalmente se desarrollarán servicios de normalización, metrología, control de calidad, de capacitación, esquemas para introducir profesionales, extensionismo tecnológico, pruebas y ensayos, laboratorios de todo tipo, información tecnológica, mejora de estadísticas, etc., todo ello incidirá en un plazo brevísimo en un incremento de la productividad y de la competitividad del país.

\section{A mediano plazo 1996-2005}

A mediano plazo, la política se orientará a la investigación aplicada y desarrollo experimental a las empresas. Se procurará que haya una especialización competente en áreas prioritarias, es decir tener verdaderas concentraciones de capacidad en áreas determinantes para el desartollo del país, que podrían ser: las relacionadas con la ecología, la agricultura y la salud, biotecnología, informática, nuevos materiales y otras, en las cuales se debería tener verdaderos expertos que solucionen los posibles. problemas.

Es necesario tener competencia concentrada en determinadas áreas, lo que se 
podrfa bacer creando centros especfficos de investigación, donde se concentra capacidad para generar tecnologia. En las empresas, es necesario ir creando capacidad para innovar, el problema famoso entre la productividad y la competitividad, el cual no se resuelve dándole tecnologías a las empresas sin que permitiendo que las empresas generen la innovación; hay excepciones donde es posible generar tecnología en centros nacionales y pasarla típicamente en el área agricola, pero el grueso de la innovación se genera en las empresas, y es a través de pequeños esfuerzos experimentales que las empresas van adquiriendo más productividad y competitividad, por lo que es importante construir dentro de las empresas la aptitud y las capacidades de hacer esto de una manera más o menos rutinaria; para ello es necesario dar ejemplo de que lo que se plantea es factible y crear un ambiente de innovación (tener acción catalítica de generar aspectos de demostración); para fomentar esto, es necesario distinguir dos tipos de empresas: las empresas con poca capacidad endógena para innovar (pequeña empresa) y las que poseen más capacidad para innovar de lo que actualmente hacen (media empresa).

\section{A largo plazo 1996-2021}

En el largo plazo es indispensable contar con un cierto "mínimo de capacidad cientffica generalizada de proporciones adecuadas al país"; lo que niega el enfioque que pretende especializar de una manera tan estrecha las competencias de un país en torno a ciertos problemas productivos. Un país que no tenga un mínimo de capacidad cientrfica generalizada podrfa caer en la trampa de la especialización tecnológica ineficaz dentro de una competencia científica generalizada. Si no hay masa crítica el país pierde eficacia. Físicamente ésto está relacionado con el desarrollo institucional de las universidades, que es donde está la falla, ya que es el desarrollo de las universidades la que determinará la capacidad de sus científicos; pero hay que invertir aunque sea modesta y continuamente en todos los niveles educativos; establecer patrones rigurosos de calidad desde el principio de la educación y promover actividades que incentiven económicamente a estos grupos de cientfficos. Un ejemplo de ello son los fondos concursales, donde se presentan propuestas de proyectos en los cuales se califican en base a su excelencia científico-técnico (donde debería determinarse el rango de calidad por extranjeros) y por su pertinencia o relevancia en el país, mezclando estas variables se puede distribuir recursos exigiendo calidad y atendiendo a las necesidades del país, lo que mantiene a la comunidad científica con más recursos, dándoles la posibilidad de acrecentar sus salarios; además sirve para llegar a complementar equipos aunque sea de una forma pequeña; esto mantiene activa a la comunidad científica y va acrecentando el interés por estas ramas logrando al final una capacidad científica generalizada.

Debe además haber una investigación básica pues es un insumo necesario para la formación de profesionales creativos, y sirve para establecer en las uni- 
veruidades masas crfiticas de recursos con capacidades. Otra forma puede ser dar becas-crédito, es decir un credito especial condonado bajo cienas circunstancias (por ejemplo, el trabajar en una institución pública o ecadturica por un tiempo corropondiente al doble del que duro la beca) y vinculado a planes institucionales. Otro medio puede ser una política de repatriación de recursos nacionales diseminados en el extranjero.

\section{Componentes de la política}

El marco de la política ha identificado los siguientes seis componentes:

1. Financiamiento al desarrollo cientffico y tecnológico

2. Fomento y gestión de la calidad y la productividad

3. Formación y capacitación de recursos humanos

4. Infraestructura de ciencia y tecnología

5. Transferencia, innovación y desarrollo tecnológico

6. Información científica y tecnológica.

\section{Seguimiento y evaluación de la política}

Para apoyar la toma de decisiones será necesario darle el seguimiento adecuado y evaluar los resultados de la política, de preferencia conforme a un conjunto de parámetros e indicadores sectoriales, institucionales, por proyecto, por personas responsables y temporalmente.

Su lógica debe responder a reorientar el proceso cuando sea pertinente, afinar prioridades, fortalecer la institucionalidad y la colaboración horizontal, de forma de mantener el rumbo adecuado y su visión, con fuerte apoyo de la acción de corto plazo.

La claridad de los planes de trabajo, acuerdos explícitos y las prioridades bien definidas son factores determinantes a ese efecto.

Estrategía de la política nacional de ciencia y tecnología

\section{Descripción básica y accionar por componentes}

\section{Financiamiento al desarrollo cientííco y tecnológico}

a. Líneas de acción:

- Creación y organización de un fondo de desarrollo científico y tecnológico.

- Establecimiento de políticas puntuales y operatividad de dicho fondo. 
- Propugnar por el financiamiento adecuado para proyectos de ciencia y lectología en el Banco Multisectorial de Inversiones y otras instituciones financieras públicas.

- Mejorar las actitudes y mecanismos en favor de la innovación tecnológica en el sistema financiero privado.

- Negociación selectiva y conveniente con las agencias multilaterales y bilaterales de financiamiento internacional.

b. Marco institucional:

- Ministerio de Hacienda

- Ministerio de Economía

- Banco Central de Reserva

- Instituciones Financieras Públicas

- ABANCA, ASOB e Instituciones Financieras Privadas

- INSAFORP.

- Organismos financieros internacionales.

c. Instrumentos:

- Fondo de desarrollo científico y tecnológico, reglamento e instructivos

- Línea(s) especial(es) de crédito

- Sistema de proyectos de ciencia y tecnología

- Contratos y fideicomisos

- Convenios de donación y préstamo

- Mecanismos de vinculación universidades-institutos tecnológicos y sistema empresarial

- Programas de investigación.

2. Fomento y gestión de la calidad y la productividad

a. Líneas de acción:

- Desarrollo y fortalecimiento del Sistema Integrado de MNCC.

- Promoción y consolidación de la cultura de calidad.

- Promoción de la reconversión productiva. 


\section{b. Marwo instituctonal}

- Ministerio de Economfa

- Comisionado Presidencial para la Modemización del Estedo

- Consejo Nacional de ciencia y Tecnología (CONACYT)

- Centro Nacional de Tecnología Agropecuaria y Forestal (CENTA)

- Gremiales empresariales, sindicales y profesionales

c. Instrumentos:

- Normalización Certificación y Acreditación

- Servicio y aseguramiento metrológico

- Reglamentos técnicos

- Comités técnicos

- Sistemas de laboratorio de pruebas y ensayos

- Premios

- Incentivos económicos fiscales y de asistencia técnica a empresas

- Vinculacón interinstitucional

- Programas de especialización recursos humanos

3. Formación y capacitación de recursos humanos

a. Líneas de acción:

- Desarrollo y fortalecimiento de la vinculación efectiva universidad-empresas

- Divulgación y promoción de la ciencia y tecnología

- Implementación de estrategias de popularización de la ciencia y tecnología.

- Capacitación de mano de obra calificada

- Integración de los contenidos de ciencia y tecnología en los programas educativos a todos los niveles de formación.

- Generación de educación y actualización continua para recursos humanos de todos los niveles

- Actualización y capacitación en planta de la mano de obra calificada

- Formación e identificación de talentos y líderes para la conducción estratégica

- Mejoramiento de programas de postgrado con excelencia en las áreas económicas, sociales y cientfficas prioritarias y estratégicas. 
- Otorgamiento de recursos especiales y extraordinarios para

- Proyectos de investigación

- Formación de recursos humanos

- Generación de recursos humanos destacados

- Mejora del equipamiento de los programas de postgrado

\section{b. Marco institucional:}

- Consejo Nacional de Ciencia y Tecnología (CONACYT)

- Universidades e institutos tecnológicos

- Instituto Salvadoreño de Formación Profesional (INSAFORP)

- Ministerio de Hacienda

- Fundaciones empresariales

- Ministerio de educación (MINED)

- Gremiales empresariales

- Colegios profesionales

c. Instrumentos:

- Museo Nacional de Ciencia y Tecnología

- Premio Nacional de Ciencia y Tecnología

- Alianzas estratégicas de financiamiento, por ejemplo Convenio de Cooperación CONACYT-INSAFORP

- Programa de incentivos a las empresas privadas para la capacitación

- Ferias de ciencias

- Becas y becas-crédito

- Fondos no reembolsables de los organismos internacionales por la capacitación de recursos humanos

- Participación de CONACYT en la selección de candidatos a becas relaciontados con ciencia y tecnología provenientes de instituciones y organismos extranjeros

- Recursos del presupuesto de la nación para financiamiento

- Coordinación CONACYT-MINED

- Convenios de gremiales consejo de Educación Superior y Consejo de Rectores de Universidades 


\section{Infraestructura de ciencia y tecnología}

\section{a. Líneas de acción:}

- Modernizar y articular la red de laboratorios de ciencia y tecnología.

- Ampliar y mejorar los servicios del Laboratorio Metrologia Legal

- Estimular a fondo la investigación cientifico-tecnológica y la creación de modernos institutos de investigación con orientación ala demanda la y tendencias del mercado.

- Reducir la fuga de personal cientifico de alto nivel hacia el exterior y estructurar un archivo maestro de personal calificado y especializado

b. Marco institucional:

- CONACYT

- CENTA

- Universidad de El Salvador

- Centro de Información Tecnológica

- Sistema SV-NET

- Institutos de investigación

c. Instrumentos:

- Red de laboratorios

- Sistema de acreditación de calidad

- Comités de normas

- Alianzas estratégicas y trabajo conjunto

5. Transferencia, innovación y desarrollo tecnológico

a. Líneas de acción:

- Desarrollo y fortalecimiento de la vinculación universidad-empresa

- Capacitación y difusión en gerencia tecnológica

- Promoción y difusión

- Integración del sistema de innovación tecnológica

- Promoción de la innovación tecnológica

- Impulsar y consolidar la cultura de emprendedores en el sistema educativo 
- Promoción y difusión de los avances nacionales en ciencia y tecnologra

- Crear y mejorar las ventajas competitivas, por producto o servicio y sectoriales

- Fortalecer la integración al proceso de globalización y penetración comercial

- Mantener y acelerar la gestión sobre el enlace permanente universidadesinstitutos tecnológicos y sistema empresarial

b. Marco institucional:

- Universidades

- Institutos tecnológicos

- CONACYT

- Centro Nacional de Tecnología y Forestal (CENTA)

- Gremiales empresariales profesionales y sindicales

- Fundaciones empresariales

- Instituto Salvadoreño de Formación Profesional (INSAFORP)

- Escuela Nacional de Agricultura (ENA)

c. Instrumentos:

- Programas de apoyo

- Programas de capacitación

- Programas de investigación

- Fondo(s) de financiamiento

- Seminarios becas

- Premios nacionales

- Ferias y programas de intercambio

- Programas de difusión

- Misiones de especialistas

- Parques tecnológicos

- Incubadoras de empresas de base tecnológica

6. Información científica y tecnológica

a. Líneas de acción:

- Establecer un sistema de indicadores básicos sobre ciencia y tecnologfa 
- Mejorar y promocionar mucho más los beneficios de la Red SV-NET

- Impulsar el uso y multiplicación de la información en la gestión de ciencia y tecnología vinculada al sistema empresarial

- Articular las redes naturales y de apoyo a la información científico-tecnológica

- Ampliar a información comercial par ala micro y pequeña empresas

b. Marco institucional:

- CONACYT

- CENTA

- Grupo SV-NET

- Comité Nacional de Informática

- Gerencia de redes regionales e internacionales

- Banco Central de Reserva, Ministerio de Hacienda y Dirección General de Estadística y Censos

- Gremiales empresariales

c. Instrumentos:

- Centro de Información Tecnológica

- Red SV-NET

- Censos y encuestas

- Revistas boletines y otras publicaciones

- Programas de capacitación

- Revista de Ciencia y Tecnología

- Redes Regionales y Hemisféricas

CONACYT, Junta Directiva, Dirección Ejecutiva y Depto. de Desarrollo Científico y Tecnológico.

San Salvador 20 de noviembre de 1996. 